Results We found extracellular mitochondrial DNA (mtDNA) and mitochondrial organelles in the plasma of patients with SLE. The concentrations of mtDNA and extracellular mitochondria were higher than in healthy individuals, and mtDNA levels correlated with that of platelet factor 4 (PF4), a marker of platelet degranulation. The majority of the detected mtDNA was associated with the extracellular mitochondrial organelle. In our in vitro and in vivo investigations, mitochondrial release by platelets required the stimulation of platelet Fc $\gamma$ RIIA, a receptor for immune complexes. Fc $\gamma$ RIIA expression in lupus-prone mice accelerated nephritis, led to the recruitment of platelets in kidneys and to the release of mitochondria in vivo. Using our mouse model with fluorescently labeled mitochondria, we confirmed platelets as a source of extracellular mitochondria driven by FcyRIIA and its co-signaling by the fibrinogen receptor $\alpha 2 b \beta 3$ in vivo.

Conclusion Our findings suggest that platelets might be a key source of mitochondrial antigens in SLE and might be a therapeutic target for treating SLE.

Acknowledgments This work was supported by a Foundation grant from the Canadian Institutes of Health Research (CIHR) (to E.B.). E.B. is recipient of a new investigator award from the CIHR and the Fonds de Recherche en Santé du Quebec (FRQS) PRF is recipient of a tier 1 Canada Research Chair on Systemic Autoimmune Rheumatic Diseases.

\section{DOES ANTIMALARIAL ADHERENCE DECREASE THE RISK OF CARDIOVASCULAR EVENTS AND MORTALITY AMONG PATIENTS WITH INCIDENT SYSTEMIC LUPUS ERYTHEMATOSUS AND RHEUMATOID ARTHRITIS? A POPULATION-BASED STUDY}

\footnotetext{
1,2M Rashedul Hoque* ${ }^{*}$ 1,3 J Antonio Avina-Zubieta, 1,3Diane Lacaille, 1,4Mary ADe Vera, ${ }^{5}$ Yi Qian, ${ }^{1,3}$ John M Esdaile, ${ }^{1,2}$ Hui Xie. ${ }^{1}$ Arthritis Research Canada, BC, Canada; ${ }^{2}$ Faculty of Health Sciences, Simon Fraser University, Canada; ${ }^{3}$ Division of Rheumatology, Department of Medicine, University of British Columbia (UBC), Canada; ${ }^{4}$ Faculty of Pharmaceutical Sciences, UBC, Canada; ${ }^{5}$ Sauder School of Business, UBC, Canada
}

10.1136/lupus-2021-lupus21century. 18

Background There is an evidence of poor adherence (ranging from $25 \%$ to $57 \%$ ) to antimalarial (AM) medications in systemic lupus erythematosus (SLE) and rheumatoid arthritis (RA). We examine the association between AM adherence and cardiovascular (CVD) events and mortality among incident SLE and RA patients, and assess if the association in SLE differs from that in RA.

Methods All patients with incident SLE or RA and incident AM use in British Columbia, Canada, between January 1997 and March 2015 were identified using provincial administrative databases. The outcomes were incident CVD events and mortality attributed to myocardial infarction, stroke or venous thromboembolism. We used marginal structural models (MSM) to estimate the effect of AM adherence on incident CVD events and mortality, accounting for potential confounders and competing events due to death unrelated to CVD. In the analysis, for each 90-days window of follow-up time, the proportion of days covered (PDC), a measure of adherence, was calculated and categorized as adherence (PDC $\geq 0.90)$, partial adherence $(0<\mathrm{PDC}<0.90)$, and non-taking $(\mathrm{PDC}=0)$ for $\mathrm{AM}$ use. The analyses were controlled for baseline demographics as well as the following sets of baseline and time-varying covariates: medication use, health resource utilization, comorbidities, and Romano adaptation of Charlson comorbidity index. Also, SLE and RA patients were analyzed separately.

Results We identified 21,114 individuals with incident SLE or RA $(3,496$ SLE; 17,618 RA patients, mean age 55.8 years, $75.9 \%$ female) with $\geq 1$ filled AM prescription. Over the mean follow-up of 8.8 and 8.6 years, $2759(14.3 \%)$ and $900(4.3 \%)$ patients experienced incident CVD events and CVD mortality, respectively. The incidence rates of CVD mortality for AM adherence, partial adherence, and non-taking were $3.10,4.68$, and 5.65 per 1000 personyears. Using MSM, the adjusted hazard ratios (aHRs) of CVD mortality obtained for AM partial adherence and adherence in SLE or RA patients were 1.08 (95\% CI: 0.91 1.31 ) and 0.51 (95\% CI: 0.42-0.62), respectively, relative to non-taking (table 1). Also, the aHR for adherence compared to partial adherence was 0.47 (95\% CI: 0.37-0.60). Findings were similar for CVD events (table 1). There was no significant difference in risk estimates between SLE and RA patients (Wald test p-values, table 1) though SLE patients

Abstract 405 Table 1 Overall risk of incident CVD events and mortality in incident SLE and RA patients during follow-up

\begin{tabular}{|c|c|c|c|c|c|c|c|c|c|}
\hline \multicolumn{5}{|c|}{ Both SLE and RA } & \multicolumn{2}{|c|}{ SLE patients } & \multicolumn{2}{|c|}{ RA patients } & \multirow{2}{*}{$\begin{array}{l}\text { Wald test } p \text {-values } \\
\text {-comparing SLE and } \\
\text { RA }\end{array}$} \\
\hline Adherence Levels & $\begin{array}{l}\text { Outcome } \\
\text { counts }\end{array}$ & $\begin{array}{l}\text { IR per } 1000 \\
\text { person-years }\end{array}$ & $\begin{array}{l}\text { IR Ratios } \\
(95 \% \mathrm{Cl})\end{array}$ & $\begin{array}{l}\text { MSM aHRs } \\
(95 \% \mathrm{Cl})\end{array}$ & $\begin{array}{l}\text { Outcome } \\
\text { counts }\end{array}$ & $\begin{array}{l}\text { MSM aHRs } \\
(95 \% \mathrm{Cl})\end{array}$ & $\begin{array}{l}\text { Outcome } \\
\text { counts }\end{array}$ & $\begin{array}{l}\text { MSM aHRs } \\
(95 \% \mathrm{Cl})\end{array}$ & \\
\hline \multicolumn{10}{|c|}{ Outcome: Incident CVD events } \\
\hline Non-taking [Reference] & 1,706 & 18.40 & 1.00 & 1.00 & 136 & 1.00 & 1,570 & 1.00 & \\
\hline Partial adherence & 527 & 16.47 & $0.90(0.81-0.99)$ & $1.02(0.92-1.13)$ & 80 & $1.17(0.88-1.56)$ & 447 & $1.01(0.91-1.13)$ & 0.37 \\
\hline Adherence & 526 & 14.00 & $0.76(0.69-0.84)$ & $0.72(0.65-0.80)$ & 83 & $0.90(0.68-1.20)$ & 443 & $0.70(0.63-0.78)$ & 0.14 \\
\hline Contrast: Adherence & & & $0.85(0.75-0.96)$ & $0.70(0.62-0.80)$ & & $0.77(0.56-1.06)$ & & $0.69(0.60-0.79)$ & 0.55 \\
\hline \multicolumn{10}{|l|}{ vs. Partial adherence } \\
\hline \multicolumn{10}{|c|}{ Outcome: CVD mortality } \\
\hline Non-taking [Reference] & 602 & 5.65 & 1.00 & 1.00 & 30 & 1.00 & 572 & 1.00 & \\
\hline Partial adherence & 165 & 4.68 & $0.82(0.70-0.98)$ & $1.08(0.91-1.31)$ & 9 & $0.61(0.29-1.28)$ & 156 & $1.13(0.94-1.37)$ & 0.06 \\
\hline Adherence & 133 & 3.10 & $0.55(0.45-0.66)$ & $0.51(0.42-0.62)$ & 16 & $0.66(0.35-1.26)$ & 117 & $0.50(0.40-0.61)$ & 0.46 \\
\hline Contrast: Adherence & & & $0.66(0.53-0.83)$ & $0.47(0.37-0.60)$ & & $1.08(0.46-2.52)$ & & $0.44(0.34-0.56)$ & 0.17 \\
\hline vs. Partial adherence & & & & & & & & & \\
\hline
\end{tabular}

Non-taking: $\mathrm{PDC}=0$, Partial adherence: $0<\mathrm{PDC}<0.90$, Adherence: $\mathrm{PDC} \geq 0.90$. Abbreviations: IR, incidence rate; aHR, adjusted hazard ratio; $\mathrm{MSM}$, marginal structural model; $\mathrm{Cl}$, confidence interval; PDC, proportion of days covered. 
had nonsignificant results, possibly due to the small sample size, especially for mortality.

The multivariable models were adjusted for baseline covariates including demographic variables (age, sex, location of residence, neighborhood income quintile), indicator of having RA or SLE (not considered for individual RA and SLE patients cases), health resource utilization (hospital visits, physician and specialist visits including rheumatologist, nephrologist, and psychiatrist visits), medication usage (statins, other cardiovascular drugs, hormone replacement therapy, glucocorticoids, anticoagulant therapy, Cox-2 inhibitors, immunosuppressive drugs), comorbidities (hypertension, chronic obstructive pulmonary disease, angina), and the Romano adaptation of the Charlson comorbidity index for administrative data. Also, the time-varying variables of health resource utilization, medication usage, Charlson comorbidity index, and comorbidities were used to calculate weights in marginal structural model.

Conclusions SLE and RA patients' adherent to AM therapy had 53\% and 30\% lower risk of CVD mortality and incident CVD events, respectively, than partially adherent patients.

\section{0 - Lupus nephritis}

\section{ANTI-LG3 ANTIBODIES CONTRIBUTE TO MICROVASCULAR LOSS AND FIBROSIS IN LUPUS NEPHRITIS}

\footnotetext{
1,2,3 Marie-Hélene Normand, 23,4Shanshan Lan, 1,2,3 Sandrine Juillard, 2,3Julie Turgeon, ${ }^{2}$ Annie Karakeussian-Rimbaud, ${ }^{2,3}$ Francis Migneault, Éric Boilard ${ }^{3,5}$ Guillaume Bollée 2,42,3,4 Marie-Josée Hébert, ${ }^{1,2,3}$ Mélanie Dieudé*. ${ }^{1}$ Département Microbiologie, Infectiologie et Immunologie, Faculté de Médecine, Université de Montréal; ${ }^{2}$ Centre de Recherche du CHUM (CRCHUM); ${ }^{3}$ Canadian Donation and Transplantation Research Program (CDTRP); ${ }^{4}$ Université de Montréal; ${ }^{5}$ Université Laval
}

\subsection{6/lupus-2021-lupus21century.19}

Background Lupus nephritis (LN) is a common and serious manifestation of systemic lupus erythematosus (SLE) and 30\% of cases, affected patients progress to end stage renal disease (ESRD). Microvascular damage is an emerging contributing factor to LN renal dysfunction leading to end stage renal disease. We have shown that vascular injury derived apoptotic exosomes can trigger SLE autoantibodies as well as autoantibodies targeting perlecan/LG3 (anti- LG3). We have also unraveled biomarkers and effector roles of anti-LG3 in kidney vascular damage in both native and transplanted kidneys. We hypothesize that anti-LG3 responses contribute to microvascular damage of importance in LN development.

Methods Longitudinal bleeds were performed on SLE prone NZB/NZWF1 and control mice. Circulating Anti-LG3 IgG levels were measured by ELISA. 26 weeks old NZB/NZWF1 mice were infused with anti-LG3 or control IgG, every second day for 3 weeks. Kidneys were harvested at sacrifice for renal histology and immunohistochemistry analyses. PTC capillary loss was evaluated with MECA-32 staining and renal interstitial fibrosis aSMA and Collagen IV staining.

Results Elevated levels of anti-LG3 are found in SLE prone mice, compared to control mice. Importantly, NZB/NZWF1 mice passively transferred with anti-LG3 exhibited significantly increased interstitial inflammation, PTC capillary loss, and renal interstitial fibrosis in the absence of glomerular abnormalities (crescents and proliferation), compared to mice transferred with control IgG.
Conclusions These observations suggest that anti-LG3 antibodies are elevated in SLE and show a tropism specific for the interstitial microvasculature, contributing to microvascular loss and fibrosis. A better understanding of the impact of these novel biomarkers and effector will improve identification, prediction, and management of LN.

\section{ENVIRONMENTAL ADAPTATION AND TISSUE INJURY IN LUPUS}

Ping-Men Chen, Joseph Craft*. Yale University

10.1136/lupus-2021-lupus21 century.20

Background Tissue injury with progressive damage is a major cause of morbidity and mortality in SLE. Yet, there is limited knowledge of the mechanistic pathways that contribute to tissue injury in lupus. We hypothesize that local environmental factors dictate effector function of tissue-infiltrating immune cells in target organs in SLE, to initiate and sustain damage, with therapeutic targeting of these maladaptive changes able to reverse damage. Our hypothesis is based upon the idea that local microenvironment naturally responds to stress or insult, such as mediated by glomerular immune complex deposition or environmental damage to the skin, with changes in the local microenvironment affecting immune-cell phenotype and function.

Methods and Results To identify tissue-adaptive pathways mediated by the local microenvironment that initiate and sustain organ injury, we dissected tissue-infiltrating $\mathrm{T}$ cells in the lupus kidney, building upon the longstanding observation that $\mathrm{T}$ effector cells are necessary for the local inflammatory response. For example, tissues become hypoxic as a common denominator as an appropriately physiologic response occurring at sites of pathogen replication or in tumors to ensure lymphocyte survival and effector function. The kidney, naturally hypoxic at the corticomedullary junction and in the medulla, becomes even more hypoxic during inflammation. We demonstrated that upon renal infiltration, $T$ cells adapt to the hypoxic environment, without exhaustion, to ensure their survival and effector capability, with remodeling of metabolic and epigenetic pathways with tissue damage driven by a hypoxia-dependent, transcriptionally regulated inflammatory gene program. Tissue hypoxia was initiated by autoantibodydependent glomerular immune complex formation, with subsequent renal entry of $\mathrm{T}$ cells that environmentally adapt with tissue-damaging effector function of a type 1 (Th1 or Tc1) phenotype. Survival was sustained by genetic and metabolic reprogramming of $\mathrm{CD}^{+}$and $\mathrm{CD}^{+}{ }^{+} \mathrm{T}$ cells, including alteration in cell death and energy utilization pathways. Selective genetic, or more practically, pharmacologic blockade of tissue-adaptive pathways dampened activated T-cell function, reversing tissue hypoxia and alleviating established damage. The same pathways are operative in human lupus nephritis.

Conclusions We demonstrate that upon infiltration into the kidney, $\mathrm{T}$ cells adapt to the local environment to ensure their survival and effector capability, with remodeling of metabolic pathways. Although beneficial to the host upon local pathogen invasion, such changes in lupus are maladaptive, leading to tissue damage. Understanding phenotypic changes in renal-infiltrating immune cells can lead to therapeutic targeting with disease amelioration. 